\title{
Nonsingular Positon Solutions of a Variable-Coefficient Modified KdV Equation
}

\author{
Yi Lin, Chuanzhong Li, Jingsong He \\ Department of Mathematics, Ningbo University, Ningbo, China \\ Email: yumeng.414@163.com
}

Received 2013

\begin{abstract}
The determinant representation of three-fold Darboux transformation for a variable-coefficient modified KdV equation is displayed based on the technique used to solve Ablowitz-Kaup-Newell-Segur system. Additionally, the nonsingular positon solutions of the variable-coefficient modified $\mathrm{KdV}$ equation are firstly discovered analytically and graphically.
\end{abstract}

Keywords: Variable-Coefficient KdV Equation; Lax Pair; Darboux Transformation; Positon; Soliton-Positon

\section{Introduction}

The differential nonlinear evolution equations (NLEEs) have been researched extensively in the past decades. Recently, investigators are shifting their focus in the NLEEs with variable coefficients as in [1]. It is found that NLEEs with variable coefficients can provide more powerful and realistic models than the ones with constant coefficients in describing more complex and real phenomenon. It is shown that solitons of NLEEs with variable coefficients can be effectively controlled through changing their variable parameters.

In this paper, we will concentrate on a variable-coefficient modified $\mathrm{KdV}$ (vc-mKdV) equation

$$
u_{t}+\alpha(t) u_{x x x}+\beta(t) u^{2} u_{x}+\delta(t) u+\gamma(t) u_{x}=0,
$$

where $\alpha(t), \beta(t), \delta(t), \gamma(t)$ are all time-dependent analytic functions.

In fact, much attention has been paid to research on different forms of vc-mKdV equations. Particularly, as long ago as 1996, K. Porsezian investigated the $\mathrm{N}$-soliton solution of a vc-mKdV equation is derived through the Hirota method in [2]. And the double-Wronskian-typed soliton of the vc-mKdV Equation (1) are constructed recently in [3].

Besides the soliton solution of NLEEs, another kind of solution called positon, is researched in lots of papers as well, such as [4-6]. As we know, positon relates very closely to soliton, i.e. the interdependence among spectral parameters of soliton gives rise to positon, so we can also call positon "degenerate soliton" [7].

This letter is organized as follows. In Section 2, we construct the detailed Darboux Transformation and its determinant representation of Equation (1). Furthermore, the nonsingular positon and soliton-positon solutions of Equation (1) are derived firstly in Section 3, a series of pictures are also displayed for the better understanding. Section 4 is devoted to the conclusions and discussions.

\section{Darboux Transformation of the vc-mKdV Equation}

\subsection{Lax pair of the ve-mKdV Equation}

It's known that the Lax pair plays a vital role in studying the integrable properties of NLEEs such as the Hamiltonian structures, conservation laws, symmetry and Darboux Transformations show in [7-10]. In the following research, we will use the lax pair presented in [3], which is constructed with the help of the Ablowitz-Kaup-Newell-Segur (AKNS) approach under the following constraint

$$
\beta(t)=6 / C_{0}^{2} \alpha(t) e^{2 \int \delta(t) d t},
$$

where $C_{0} \neq 0$ is an arbitrary constant. We will choose $C_{0}=1$ in this paper for simplicity.

Then the linear eigenvalue problems for Equation (1) can be expressed as

$$
\Phi_{x}=M \Phi, \Phi_{t}=N \Phi .
$$

where $\Phi=\left(f_{1}, f_{2}\right)^{T}, T$ denotes the transpose of the matrix, and $M$ and $N$ are shown as follows:

$$
\begin{aligned}
& M=\left(\begin{array}{cc}
\lambda & e^{\int \delta(t) d t} u \\
e^{\int \delta(t) d t} v & -\lambda
\end{array}\right), N=\left(\begin{array}{cc}
A & B \\
C & -A
\end{array}\right), \\
& A=-4 \alpha(t) \lambda^{3}-\lambda\left[\gamma(t)-2 e^{2 \int \delta(t) d t} \alpha(t) u v\right] \\
& +\alpha e^{2 \int \delta(t) d t}\left(u_{x} v-v_{x} u\right) \\
& \text { with } \\
& B=-e^{\int \delta(t) d t}\left[4 \lambda^{2} \alpha(t) u+2 \lambda \alpha(t) u_{x}+\alpha(t) u_{x x}\right. \\
& \left.-2 e^{2 \int \delta(t) d t} \alpha(t) u^{2} v+\gamma(t) u\right] \\
& C=e^{\int \delta(t) d t}\left[-4 \lambda^{2} \alpha(t) v+2 \lambda \alpha(t) v_{x}-\alpha(t) v_{x x}\right. \\
& \left.+2 e^{2 \int \delta(t) d t} \alpha(t) u v^{2}-\gamma(t) v\right]
\end{aligned}
$$


The spectral parameter $\lambda$ is independent of $x$ and $t$. With constraint (2), it is easy to prove that the compatibility condition (also called the zero curvature equation) of Equations (3) and (4).

$$
M_{t}-N_{x}+[M, N]=0
$$

gives rise to Equation (1) for $u=-v$ by direct computation. The bracket represents the usual matrix commutator, and the Lax pair (3) can guarantee the complete integrability of Equation (1).

\subsection{Three-fold Darboux Transformation for the vc-mKdV Equation}

First of all, we need to introduce the eigenfunctions which stasify the Lax pair (3)

$$
\Phi_{k}=\Phi\left(\lambda_{k}\right)=\left(\begin{array}{l}
f_{k 1} \\
f_{k 2}
\end{array}\right)
$$

of all the eigenvalues $\lambda_{k}, k=1,2, \cdots, 6$, we let $\lambda_{k} \neq \lambda_{m}$ if $k \neq m$ and $\lambda_{k} \neq \lambda$. Additionally, the eigenfunctions are linearly independent i.e. $\Phi_{k}$ and $\Phi_{m}$ are linearly independent if $k \neq m$. According to the knowledge of DT, with the help of Cramer's rule and iterative computations, the explicit new solutions $u^{[3]}$ and $v^{[3]}$ of the vc-mKdV Equation (1) can be derived as

$$
\begin{aligned}
& u^{[3]}=u-2 U_{6} / \sigma W_{6}, \\
& v^{[3]}=v+2 V_{6} / \sigma W_{6} .
\end{aligned}
$$

where

$$
\begin{aligned}
U_{6} & =\left|\begin{array}{llllll}
f_{11} & f_{12} & \lambda_{1} f_{11} & \lambda_{1} f_{12} & \lambda_{1}^{2} f_{11} & \lambda_{1}^{3} f_{11} \\
f_{21} & f_{22} & \lambda_{2} f_{21} & \lambda_{2} f_{22} & \lambda_{2}^{2} f_{21} & \lambda_{2}^{3} f_{21} \\
f_{31} & f_{32} & \lambda_{3} f_{31} & \lambda_{3} f_{32} & \lambda_{3}^{2} f_{31} & \lambda_{3}^{3} f_{31} \\
f_{41} & f_{42} & \lambda_{4} f_{41} & \lambda_{4} f_{42} & \lambda_{4}^{2} f_{41} & \lambda_{4}^{3} f_{41} \\
f_{51} & f_{52} & \lambda_{5} f_{51} & \lambda_{5} f_{52} & \lambda_{5}^{2} f_{51} & \lambda_{5}^{3} f_{51} \\
f_{61} & f_{62} & \lambda_{6} f_{61} & \lambda_{6} f_{62} & \lambda_{6}^{2} f_{61} & \lambda_{6}^{3} f_{61}
\end{array}\right|, \\
V_{6} & =\left|\begin{array}{llllll}
f_{11} & f_{12} & \lambda_{1} f_{11} & \lambda_{1} f_{12} & \lambda_{1}^{2} f_{12} & \lambda_{1}^{3} f_{12} \\
f_{21} & f_{22} & \lambda_{2} f_{21} & \lambda_{2} f_{22} & \lambda_{2}^{2} f_{22} & \lambda_{2}^{3} f_{22} \\
f_{31} & f_{32} & \lambda_{3} f_{31} & \lambda_{3} f_{32} & \lambda_{3}^{2} f_{32} & \lambda_{3}^{3} f_{32} \\
f_{41} & f_{42} & \lambda_{4} f_{41} & \lambda_{4} f_{42} & \lambda_{4}^{2} f_{42} & \lambda_{4}^{3} f_{42} \\
f_{51} & f_{52} & \lambda_{5} f_{51} & \lambda_{5} f_{52} & \lambda_{5}^{2} f_{52} & \lambda_{5}^{3} f_{52} \\
f_{61} & f_{62} & \lambda_{6} f_{61} & \lambda_{6} f_{62} & \lambda_{6}^{2} f_{62} & \lambda_{6}^{3} f_{62}
\end{array}\right| .
\end{aligned}
$$

Next, it's very easy to turn out that if $\mathrm{f}_{\mathrm{k}+11}=-\mathrm{f}_{\mathrm{k} 2}$, $\mathrm{f}_{\mathrm{k}+12}=\mathrm{f}_{\mathrm{k} 1}, \quad \lambda_{k+1}=-\lambda_{k} \quad(k=1,3,5)$, then $u^{[3]}=-v^{[3]}$ will be realized, so the three-fold DT of the vc-mKdV equation (1) is accomplished completely. Additionally, we can get the analogous expressions of $u^{[2]}$ and $v^{[2]}$ when $\mathrm{k}=1,2,3,4$, so the two-soliton can be arrived as well.

\section{Positon and Soliton-positon Solutions of the vc-mKdV Equation}

Matveev expounded "positon" in [4] for the KdV equations. As we know, most of the known positon solutions are singular. For various important integrable systems such as the $\mathrm{KdV}$ equations and the $\mathrm{mKdV}$ equations, there is no nonsingular positon found, though [6] gives the nonsingular positon for the coupled $\mathrm{KdV}$ system. In this section, the positon solutions of the vc-mKdV Equation (1) will be displayed for the first time using the result obtained in section 2. It's happy that they are nonsingular.

Choosing the zero seed solution of Equation (1), Equation (3) is solved by

$$
\begin{gathered}
\Phi_{k}=\Phi\left(\lambda_{k}\right)=\left(\begin{array}{l}
f_{k 1} \\
f_{k 2}
\end{array}\right), \\
f_{k 1}=e^{\int\left(-4 \lambda_{k}^{3} \alpha(t)-\lambda_{k} \gamma(t)\right) d t+\lambda_{k} x}, \\
f_{k 2}=e^{\int\left(4 \lambda_{k}^{3} \alpha(t)+\lambda_{k} \gamma(t)\right) d t-\lambda_{k} x} .
\end{gathered}
$$

with $\lambda_{k}(k=1,2, \cdots, 6)$ are arbitrary spectral Parameters.

In the construction of two-soliton solutions, if the second spectral parameter $\lambda_{3}$ is colse to the first spectral parameter $\lambda_{1}$, then the one-positon solution of vc$\mathrm{mKdV}$ Equation (1) can be generated by doing the Taylor expansion of the wave function (8b) to the first order up to $\lambda_{1}$. That is to say, firstly, taking $\lambda_{3}=\lambda_{1}+\epsilon$ in the used eigenvalue of the two-fold DT. Secondly, using the Taylor expansion of $f_{3}$ and $f_{4}$ up to the first order of $\epsilon$ in terms of $\lambda_{1}$. Finally, substituting these manipulations into the two-fold DT, we get the one-positon solution in the form of

$$
\begin{aligned}
u_{p o s}= & e^{-\int \delta(t) d t}\left[8 \lambda _ { 1 } \left(-2 \lambda_{1} h_{1} \sinh \left(P_{1}\right)-2 \lambda_{1} x \sinh \left(P_{1}\right)\right.\right. \\
& \left.\left.+\cosh \left(P_{1}\right)\right)\right] /\left(1+8 \lambda_{1}^{2}\left(h_{1}+x\right)^{2}+\cosh \left(2 P_{1}\right)\right)(9)
\end{aligned}
$$

with

$$
\begin{gathered}
h_{1}=\int\left(-12 \alpha(t) \lambda_{1}^{2}-\gamma(t)\right) d t, \\
P_{k}=2 \int\left(-4 \lambda_{k}^{3} \alpha(t)-\lambda_{k} \gamma(t)\right) d t+2 \lambda_{k} x, \quad k=1,3
\end{gathered}
$$

From Equation (9), we can know that $\delta(t)$ will change the amplitude of the positon solution, just as the plot (a) in Figure 1 vividly shown. What's more, there is no zero point in the denominator of Equation (9), that means this one-positon solution is nonsingular. Positons of $\mathrm{KdV}$ are defined as long-range analogues of solitons and are slowly decreasing, oscillating solutions in [5]. From Figure 1, it is clear that two peaks in one-positon separate according to a different way in the two soliton.

Using the same method in the process of the positon above, fixing $\lambda_{5}$, and calculating the limit $\lambda_{3} \rightarrow \lambda_{1}$, we obtain the soliton-positon solution of vc-mKdV Equation 
(1). Letting $\lambda_{3}=\lambda_{1}+\epsilon, \lambda_{5}=\lambda_{1}+\epsilon$, and using the Taylor expansion of $f_{3}$ and $f_{4}$ up to the first order, $f_{5}$ and $f_{6}$ to the second order of $\epsilon$ in terms of $\lambda_{1}$, we get the two-positon solution of vc-mKdV Equation (1). Here the specific expressions of the soliton-positon and two-positon solutions aren't given out for saving space.

Comparing (a) in Figure 2 with (a) in Figure 3, we can clearly see that two parts of the soliton-positon are propagating in a close way, and the third one is independent, while each part of the two-positon will separate finally during their propagating, but the separation velocity is extraordinary slow. Comparming the plots (b) in Figures 1-3, it's easy to learn that the presences of solutions are changeable by choosing the variable coefficients $\delta(t), \alpha(t), \gamma(t)$ to be different functions of $t$. Particularly, from Figures 2 and Figure 3, we know that the soliton-positon and two-soliton solutions are nonsingular as well.

\section{Conclusions and Discussions}

In this paper, considering the vc-mKdV Equation (1), which is interesting both physically and mathematically, we derived its three-fold Darboux transformation in the form of determinant. Then the positon solutions, which have never been discovered in other papers are obtained.

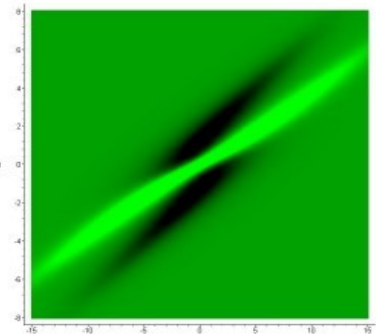

(a)

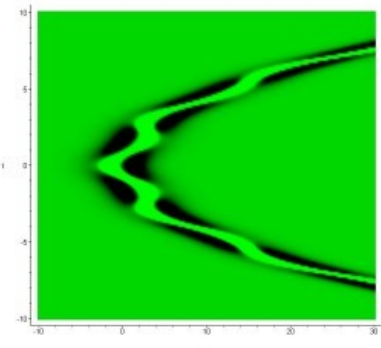

(b)
Figure 1. The dendity plots of one-positon of the vc-mKdV Equation (1) with (a) $\lambda_{1}=0.5, \delta=0.1 t, \alpha=1, \gamma=1$; (b) $\lambda_{1}=0.6, \delta=0, \alpha=\sin (2 t), \gamma=t$.

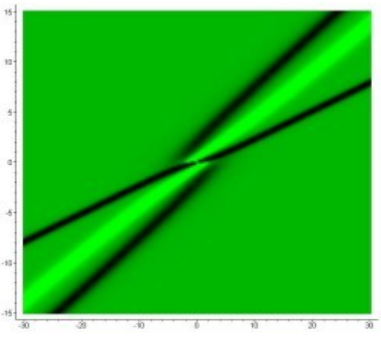

(a)

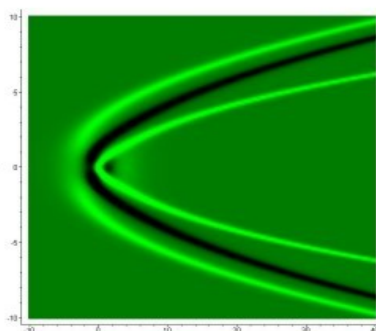

(b)
Figure 2. The dendity plots of soliton-positon of the vc-mKdV Equation (1) with $\lambda_{1}=-0.5, \lambda_{5}=-1, \delta=0, \alpha=1$, $\gamma=1$; (b) $\lambda_{1}=0.51, \lambda_{5}=0.71, \delta=0, \alpha=t, \gamma=0$.

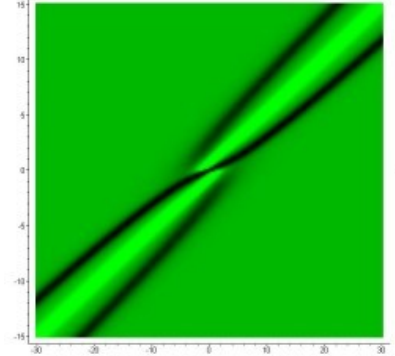

(a)

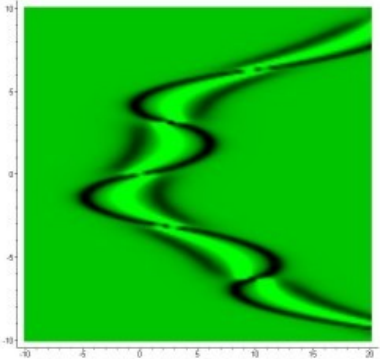

(b)
Figure 3. The dendity plots of two-positon of the vc-mKdV Equation (1) with (a) $\lambda_{1}=\mathbf{- 0 . 5}, \delta=0, \alpha=1, \gamma=1$; (b) $\lambda_{1}=-0.8$, $\delta=0, \alpha=\cos (t), \gamma=0.5 t$.

Specifically, the one-positon, soliton-positon and twopositon solutions are all nonsingular. From the Figures 1-3, it's interesting to obsrerve that these solutions exhibit the following novelty: when the variable coefficients depend on $t$, their profiles are changeful and the orbits are quite flexible rather than a straight line. We sincerely hope that these will be of use in the future study.

\section{Acknowledgements}

This work is supported by the NSF of China under Grant Nos. 10971109, 11201251 and K. C. Wong Magna Fund in Ningbo University. Jingsong He is also supported by Natural Science Foundation of Ningbo under Grant No. 2011A610179.

\section{REFERENCES}

[1] V. N. Serkin and A. Hasegawa, "Novel Soliton Solutions of the Nonlinear Schrödinger Equation Model," Physical Review Letters, Vol. 85, No. 21, 2000, pp. 4502-4505. doi:10.1103/PhysRevLett.85.4502

[2] Y. Zhang, J. B. Li and Y. N. Lv, "The Exact Solution and Integrable Properties to the Variable-Coefficient Modified Korteweg-de Vries Equation," Annals of Physics, Vol. 323, No. 12, 2008, pp. 3059-3064. doi:10.1016/j.aop.2008.04.012

[3] L. Wang, Y. T. Gao and F. H. Qi, "N-fold Darboux Transformation and Double-Wronskian-typed Solitonic Structures for a Variable-Coefficient Modified Kortweg-de Vries Equation," Annals of Physics, Vol. 327, No. 8, 2012, pp. 1974-1988. doi:10.1016/j.aop.2012.04.009

[4] V. B. Matveev, "Positon-Politon and Soliton-Positon Collisions: KdV case," Physical Letters A, Vol. 166, No. 3-4,1992, pp. 209-212. doi:10.1016/0375-9601(92)90363-Q

[5] V. B. Matveev and Positons, "Slowly Decreasing Analogues of Solitons," Theoretical and Mathematical Physics, Vol. 131 No. 1, 2002, pp. 483-497. doi:10.1023/A:1015149618529

[6] H. C. Hu, B. Tong and S. Y. Lou, "Nonsingular Positon 
and Complexi-Ton Solutions for the Coupled KdV System," Physics Letters A, Vol. 351, No. 6, 2006, pp. 403-412. doi:10.1016/i.physleta.2005.11.047

[7] J. S. He, H. R. Zhang, L. H. Wang, K. Porsezian and A. S. Fokas, "A Generating Mechanism for Higher Order Rogue Waves," 2013.

[8] J. S. He, L. Zhang, Y. Cheng and Y. S. Li, "Determinant Representation of Darboux Transformation for the AKNS System," Science in China Series A: Mathematics, Vol. 49, No. 12, 2006, pp. 1867-1878. doi:10.1007/s11425-006-2025-1
[9] K. Porsezian and K. Nakkeeran, "Optical Solitons in Presence of Kerr Dispersion and Self-Frequency Shift," Physical Review Letters, Vol. 76, No. 21, 1996, pp. 3955-3958. doi:10.1103/PhysRevLett.76.3955

[10] M. J. Ablowitz, D. J. Kaup, A .C. Newell, H. Seger, "Nonlinear-Evolution Equations of Physical Significance," Physical Review Letters, Vol. 31, No. 2, 1973, pp. 125-127. doi:10.1103/PhysRevLett.31.125

[11] H. H. Chen and C. S. Liu, "Soliton in Nonuniform Media," Physical Review Letters, Vol. 37, No.11, 1976, pp. 693-697. doi:10.1103/PhysRevLett.37.693 